\title{
Zeta Function Continuation and the Casimir Energy on Odd and Even Dimensional Spheres
}

\author{
Brian P. Dolan ${ }^{1,2}$ and Charles Nash ${ }^{1,2}$ \\ 1 Department of Mathematical Physics, St. Patrick's College, Maynooth, Ireland \\ 2 School of Theoretical Physics, Dublin Institute for Advanced Studies, 10 Burlington Road, \\ Dublin 4, Ireland
}

Received July 17, 1991; in revised form January 17, 1992

\begin{abstract}
The zeta function continuation method is applied to compute the Casimir energy on spheres $S^{N}$. Both odd and even dimensional spheres are studied. For the appropriate conformally modified Laplacian $\Delta$ the Casimir energy $\mathscr{E}$ is shown to be finite for all dimensions even though, generically, it should diverge in odd dimensions due to the presence of a pole in the associated zeta function $\zeta_{\Delta}(s)$. The residue of this pole is computed and shown to vanish in our case. An explicit analytic continuation of $\zeta_{\Delta}(s)$ is constructed for all values of $N$. This enables us to calculate $\mathscr{E}$ exactly and we find that the Casimir energy vanishes in all even dimensions. For odd dimensions $\mathscr{E}$ is never zero but alternates in sign as $N$ increases through odd values. Some results are also derived for the Casimir energy of other operators of Laplacian type.
\end{abstract}

\section{Introduction}

The treatment of relativistic quantum fields in curved spaces is a notoriously difficult problem which has only yielded partial results to date. In particular the evaluation of the energy momentum tensor for free fields has an involved and somewhat chequered history (for a review see Birrell and Davies [1]). A particularly useful tool in this regard is the heat equation for an elliptic differential operator $P$ and the corresponding zeta function $\zeta_{P}(s)$ (Gilkey [2]). The usefulness of the heat equation for the determination of effective action in quantum field theory was emphasised in Hawking [3]. An explicit evaluation of the zeta function is only possible on spaces with a high degree of symmetry and it is worthwhile examining such situations in detail in the hope of obtaining insights into its more general properties. For example the zeta function regularisation method was used by Dowker and Banach [4] for an evaluation of the Casimir energy on $S^{3}$.

In this paper the zeta function will be explicitly evaluated at the particular value of its argument $s=-1 / 2$ and some of its properties will be examined for a class of elliptic operators on $N$-dimensional spheres. The value $s=-1 / 2$ is important because it yields the Casimir energy of $S^{N}$. We shall use the $S O(N+1)$ symmetric metric on the sphere with radius $a$, and the elliptic operator considered will be the 
modified Laplace operator acting on massless scalar fields, $\phi$,

$$
\Delta \phi=\left(-\nabla^{2}+\xi R\right) \phi=0,
$$

where $R=N(N-1) / a^{2}$ is the Ricci scalar and $\xi$ a constant. It will be shown that for even dimensional spheres zeta function regularisation can be used to obtain the Casimir energy for any value of the parameter $\xi$. For the particular value

$$
\xi=\frac{1}{4}\left(\frac{N-1}{N}\right),
$$

which corresponds to conformal coupling in $N+1$ dimensions, the Casimir energy vanishes. It is a maximum, as a function of $\xi$, if $N=2 k+2$ and a minimum if $N=2 k$ for integral $k$. For odd dimensional spheres zeta function regularisation cannot in general be used to obtain the Casimir energy, due to the presence of a pole at $s=-1 / 2$. However, we shall show that for the particular choice (1.2) of conformal coupling in $N+1$ dimensions the residue of this pole vanishes giving a finite value for the Casimir energy. This value alternates in sign being positive for $N=2 k+3$ and negative for $N=2 k+1$.

It will also be shown that, if the zeta function regularisation method can be used at all to obtain the Casimir energy on $S^{N}$, then it is necessary that the trace anomaly of the energy-momentum tensor in a related $N+1$ dimensional Euclidean signature space-time, with topology $\mathbf{R} \times S^{N}$, must also vanish. This will allow us to evaluate the entire regularised energy-momentum in the $N+1$ dimensional spacetime. In particular, we shall show that the regularised energy-momentum tensor vanishes if $N+1$ is odd and the conformal coupling (1.2) is used. The special properties of the value (1.2) for $\xi$ are related to the fact that the eigenvalues of the operator (1.1) are perfect squares for this value and only for this value.

The layout of the paper will be as follows. In Sect. 2 we give a brief review of the zeta function and the heat equation, and its use in calculating Casimir energies. We also derive the condition that the trace anomaly must vanish in $N+1$ dimensions in order that the zeta function regularisation technique be applicable for the evaluation of the Casimir energy in $N$ dimensions, and show how this allows the entire energy-momentum tensor to be evaluated from a knowledge of the Casimir energy.

In Sect. 3 we discuss odd dimensional spheres, and show that $\xi$ is not arbitrary, but zeta function regularisation only works for the operator (1.1) with discrete values of $\xi$. Section 4 will then treat the case of even dimensional spheres, and it will be proven that the Casimir energy, and hence the regularised energy-momentum tensor, vanishes for the specific choice (1.2) of $\xi$. Section 5 discusses the situation when $\xi$ is not given by (1.2). Finally our concluding remarks are in Sect. 6 .

\section{The Zeta Function and the Heat Equation}

We shall first briefly review some properties of the heat equation and the zeta function for elliptic differential operators on a $N$-dimensional compact manifold without boundary $\mathscr{M}$ with Riemannian metric $g$. Given such an operator $P$ (e.g. (1.1) above) with normalised eigenfunctions $\phi_{n}(x)$ and positive eigenvalues $\lambda_{n}$,

$$
P \phi_{n}(x)=\lambda_{n} \phi_{n}(x)
$$


the zeta functions for $\Delta$ is defined by

$$
\zeta_{P}(s)=\sum_{n=0}^{\infty} \frac{1}{\lambda_{n}^{s}}
$$

Provided the eigenvalues $\lambda_{n}$ increase with $n$, this sum will converge for $\operatorname{Re}(s)$ sufficiently large. For more general values of $s$ analytic continuation is used to define $\zeta_{P}(s)$; it then becomes a meromorphic function of $s$ with only simple poles at certain rational values of $s$ as explained below.

$\zeta_{\Delta}(s)$ is important for the calculation of Casimir energies on compact manifolds which are space-like sections of $N+1$ dimensional space time. Since the eigenvalues of the operator (1.1) are the possible energies squared of the quantum field, $\lambda_{n}=\omega_{n}^{2}$ and the Casimir energy, $\mathscr{E}$, is the sum of the zero point energies of the quantum field, we have

$$
\mathscr{E}=\frac{1}{2} \sum_{n=0}^{\infty} \hbar \omega_{n}=\frac{1}{2} \hbar \zeta_{\Delta}(-1 / 2) .
$$

Of course this sum is formally infinite and must be regularised. There are various methods of achieving this and analytic continuation of the zeta function is one such method.

In order to gain more insight into the analytic structure of $\zeta_{P}(s)$ we introduce the heat kernel, $K(x, y ; \tau)$, defined by the differential equation

$$
\frac{\partial}{\partial \tau} K(x, y ; \tau)+P K(x, y ; \tau)=0
$$

where $0 \leqq \tau<\infty$, together with the boundary condition $K(x, y ; 0)=\delta(x, y)$. ( $P$ acts only on $x$ in Eq. (2.4). An explicit solution of (2.4) is given by

$$
K(x, y ; \tau)=\sum_{n=0}^{\infty} \exp \left[-\lambda_{n} \tau\right] \phi_{n}(x) \phi_{n}(y) \text {. }
$$

It can be shown, DeWitt [5], that $K(x, y ; \tau)$ has the asymptotic expansion for small $\tau$,

$$
K(x, y ; \tau)=\frac{1}{(4 \pi \tau)^{N / 2}} \sqrt{\operatorname{det}\left(\frac{\partial^{2} \sigma(x, y)}{\partial x^{i} \partial y^{j}}\right)} \exp \left[-\frac{\sigma(x, y)}{2 \tau}\right] \sum_{n=0}^{\infty} a_{n}(x, y) \tau^{n},
$$

where $\sigma(x, y)$ is the geodesic distance between $x$ and $y$ and $a_{n}(x, y)$ are functions which depend only on the Riemann tensor and its covariant derivatives on $\mathscr{M}$. The integrated heat kernel, $Z(\tau)$, is defined by

$$
Z(\tau)=\int_{\mathscr{M}} K(x, x ; \tau) \sqrt{g} d^{N} x
$$

and has the asymptotic expansion

$$
Z(\tau)=\frac{1}{(4 \pi \tau)^{N / 2}} \sum_{n=0}^{\infty} A_{n} \tau^{n}
$$

where $A_{n}=\int_{\mathscr{M}} a_{n}(x, x) \sqrt{g} d^{N} x$. The functions $a_{n}(x, x)$ can be defined recursively in $n$ and are given up to $n=3$ in Gilkey [2]. $a_{4}(x)$ are given in Amsterdamski et al. 
[10]; cf. also, in general, Avramidi $[11,12]$. For future reference, the first three $a_{n}$ for elliptic operators of the type (1.1) are

$$
\begin{aligned}
& a_{0}=0 \\
& a_{1}=\left(\frac{1}{6}-\xi\right) R, \\
& a_{2}=\frac{1}{180}\left\{R_{i j k l} R^{i j k l}-R_{i j} R^{i j}+30(1-6 \xi)^{2} R^{2}+(30 \xi-6)\left(\nabla^{2}\right) R\right\},
\end{aligned}
$$

where $R_{i j k l}$ is the Riemann tensor on $\mathscr{M}$ and $R_{i j}$ the Ricci tensor. An important property of the $a_{n}$ is that the numerical coefficients in (2.9) are independent of the dimension of $\mathscr{M}$. The importance of $Z(\tau)$ for the zeta function is that $\zeta_{P}(s)$ can be expressed as a Mellin transform

$$
\zeta_{P}(s)=\frac{1}{\Gamma(s)} \int_{0}^{\infty} \tau^{s-1} Z(\tau) d \tau .
$$

Given this expression, it is easy to isolate the poles in $\zeta_{P}(s)$ since

$$
\zeta_{P}(s)=\frac{1}{\Gamma(s)} \int_{0}^{1} \tau^{s-1} Z(\tau) d \tau+\frac{1}{\Gamma(s)} \int_{1}^{\infty} \tau^{s-1} Z(\tau) d \tau .
$$

Due to the exponential fall off of $Z(\tau)$ for large $\tau$, it is clear that the second term in the above expression is perfectly finite as a function of complex $s$. Inserting the asymptotic expansion (2.8) in (2.11) gives

$$
\zeta_{P}(s)=\frac{1}{(4 \pi)^{N / 2} \Gamma(s)} \sum_{n=0}^{\infty} \frac{A_{n}}{\left(n+s-\frac{N}{2}\right)}+\text { finite part } .
$$

Thus $\zeta_{P}(s)$ has a simple pole whenever $s=N / 2-n$ for non-negative $n$, except at $s=0$ where any possible pole is cancelled by that of $\Gamma(s)$. The residue of the pole is given by

$$
\frac{A_{N / 2-s}}{(4 \pi)^{N / 2} \Gamma(s)}
$$

Note that, for $s=0$, the pole in $\Gamma(s)$ means that the second term in (2.11) vanishes and so

$$
\zeta_{P}(0)=\frac{A_{N / 2}}{(4 \pi)^{N / 2}} .
$$

Thus a knowledge of $A_{N / 2}$ allows us to determine $\zeta_{P}(0)$ without any further information.

Now, in order to determine the Casimir energy on $\mathscr{M}$ we must set $s=-1 / 2$ and we have a pole with non-zero residue if $A_{\frac{N}{2}+\frac{1}{2}} \neq 0$. If $N$ is even then $A_{\frac{N}{2}+\frac{1}{2}}=0$ automatically and zeta function regularisation can always be used to determine the Casimir energy on $\mathscr{M}$. If $N$ is odd, zeta function regularisation cannot be used unless $A_{\frac{N}{2}+\frac{1}{2}}=0$, and this must be checked in each specific instance. The generic presence of a pole at $s=-\frac{1}{2}$ when the dimension of space is odd is reflected in other regularisation procedures. For instance, the regularised energy momentum tensor on space-times with the topology of space being $\mathbf{R}^{3} \times S^{N}$ was calculated in 
Candelas and Weinberg [6] and Birmingham and Sen [7] and found to be finite only if $N$ had the correct parity.

These conditions for the successful implementation of the zeta function regularisation method can be related to the trace anomaly of the scalar field in $N+1$ dimensions. Hawking [3] has shown that, in $N+1$ space-time dimensions with Euclidean signature, the energy-momentum tensor of conformally coupled quantum fields develops a trace anomaly

$$
T_{\mu}^{\mu}=\zeta_{\Delta_{N+1}}(0)
$$

where the subscript $N+1$ on $\Delta$ is to clarify the fact that the zeta function here is the one appropriate to the elliptic operator for quantum fields in $N+1$ space-time dimensions $(\mu=0, \ldots, N)$. In particular suppose we have a massless scalar field, in $N+1$ dimensions with metric

$$
d s^{2}=d t^{2}+g_{i j} d x^{i} d x^{j}
$$

on $\mathbf{R} \times S^{N}$ which obeys the equation

$$
\Delta_{N+1} \phi=\left(-\frac{\partial^{2}}{\partial t^{2}}-\nabla^{2}+\xi R\right) \phi=0,
$$

(here $g_{i j}$ is the $t$-independent $S O(N+1)$ symmetric metric ${ }^{1}$ on $S^{N}$ with $i, j=1, \ldots, N)$. Then the trace anomaly is

$$
T_{\mu}^{\mu}=\zeta_{\Delta_{N+1}}(0)=\frac{A_{\frac{N}{2}+\frac{1}{2}}}{(4 \pi)^{\left(\frac{N}{2}+\frac{1}{2}\right)}} .
$$

As mentioned earlier, the numerical coefficients in $a_{n}(x, x)$ do not depend on $N$ and it is easily seen (from (2.9) with $-\nabla^{2}$ replaced with $-\partial^{2} / \partial t^{2}-\nabla^{2}$ and noting that $R_{\mu v \rho \sigma}$ is independent of $t$ ) that $a_{1}, a_{2}$ and $a_{3}$ are identical both for the metric $g_{i j}$ on $S^{N}$ and for the metric (2.16) on $\mathbf{R} \times S^{N}$. This is a special property of the trivial product structure of the metric (2.16) and would not be true of a more general metric. It persists for the higher $a_{n}(x), n>2$. Hence we see that the condition $\mathrm{A}_{\frac{N}{2}+\frac{1}{2}}=0$ for the pole at $s=-\frac{1}{2}$ in the $N$-dimensional zeta function on $S^{N}$ to vanish is identical to the condition that the trace anomaly vanishes in $N+1$ dimensions with the metric (2.16).

With this observation, the entire renormalised energy-momentum tensor can be determined on $\mathbf{R} \times S^{N}$. Let $V$ be the volume of $S^{N}$ and $\rho=\mathscr{E} / V$ be the Casimir energy density. Then, since the trace anomaly vanishes and the spatial part of the energy-momentum tensor must be $S O(N+1)$ symmetric, we must have

$$
T_{\nu}^{\mu}=\operatorname{diag}\left(\rho,-\frac{\rho}{N}, \ldots,-\frac{\rho}{N}\right) .
$$

\footnotetext{
${ }^{1}$ Strictly speaking one should use a finite segment of the real line, rather than $\mathbf{R}$ itself, in order to have a compact $N+1$ dimensional manifold, and invoke the heat equation on a manifold with boundary, (Moss [8], McAvity and Osborn [9]). However the form of the metric (2.16) is so simple that all the boundary terms vanish, either because the second fundamental form for the boundary vanishes or because the opposite orientations of the two copies of $S^{N}$ at either end cancel. Thus there is no need to introduce any boundary terms and we can use the whole real line without ambiguity
} 


\section{The Casimir Energy of Odd Dimensional Spheres}

We now turn our attention to odd dimensional spheres $N=2 p-1$. For odd dimensional spheres, the residue of the pole in $\zeta_{\Delta}(s)$ at $s=-1 / 2$ is proportional to the coefficient $A_{p}$, and we must demand that $A_{p}=0$ in order that zeta function regularisation yield a finite answer for the Casimir energy. $A_{p}$ can be calculated explicitly for low values of $p$, using the formulae (2.9) and the explicit form for the Riemann and Ricci tensors on $S^{N}$ in an orthonormal basis,

$$
R_{i j k l}=\frac{1}{a^{2}}\left(\delta_{i k} \delta_{j l}-\delta_{i l} \delta_{j k}\right), \quad R_{i j}=\frac{1}{a^{2}}(N-1) \delta_{i j},
$$

and noting that $R_{i j k l}$ is covariantly constant.

For $S^{3}$ we find from (2.9),

$$
a_{2}=6(1-6 \xi)^{2} \text {. }
$$

Thus $A_{2}=0$ if and only if $\xi=1 / 6$, which is the value for conformal coupling in four dimensions and the value which makes the eigenvalues of the elliptic operator (1.1) into perfect squares.

For $S^{5}$ the expression for $a_{3}$ can be found in Gilkey [2] (it is $a_{6}$ in Gilkey's notation). We do not reproduce it here, but merely quote the result for the operator (1.1),

$$
a_{3}=\frac{16}{3}(5 \xi-1)^{2}(1-10 \xi) .
$$

Thus zeta function regularisation works for two values of $\xi, \xi=1 / 5$ and $\xi=1 / 10$. The former is the value that gives conformal coupling in six dimensions.

For $S^{7}$ the expression for $a_{4}$ can be found in Amsterdamski et al. [10] (it is $b_{8}$ in their notation). Again we merely quote the result for the operator (1.1),

$$
a_{4}=\frac{3}{40}\left(1728720 \xi^{4}-1152480 \xi^{3}+277144 \xi^{2}-28056 \xi+981\right) .
$$

If we define $x=14 \xi / 3$, since $3 / 14$ is the value of $\xi$ which gives conformal coupling in eight dimensions, this simplifies to

$$
a_{4}=\frac{27}{40}(x-1)^{2}\left(405 x^{2}-450 x+109\right) .
$$

This polynomial has four roots, and we find the critical values of $\xi$ to be

$$
\xi=\frac{3}{14}, \frac{1}{21}\left(\frac{5}{2} \pm \frac{2 \sqrt{5}}{5}\right) .
$$

Again, for our purposes, the value $3 / 14$ is the most interesting.

To the authors' knowledge there is no explicit formula for $a_{5}$ in the literature (and even if there were, we would baulk at the calculation for $S^{9}$ ), but clearly the condition that the pole vanishes for $S^{N}$ will give a polynomial in $\xi$ of order $p$ and it is tempting to conjecture that there will always be a root at the special value $\xi=(N-1) / 4 N$ which gives conformal coupling in $N+1$ dimensions and simultaneously renders perfect squares for the eigenvalues of the elliptic operator. To 
prove this conjecture, we shall now abandon differential geometric techniques and turn to a direct construction of the analytic continuation of the zeta function.

First of all let $I$ denote the identity operator so that the operator $\Delta$ is of the form

$$
\Delta=-\nabla^{2}+\lambda I, \quad \text { where } \lambda=\xi R \equiv \frac{\xi N(N-1)}{a^{2}} .
$$

For convenience we now set the radius $a$ of the sphere to unity and we also implement our "conformality condition" $\xi=(N-1) / 4 N$. Hence for $\lambda$ we now have

$$
\lambda=\frac{(N-1)^{2}}{4}
$$

Next note that the ordinary Laplacian $-\nabla^{2}$ on $S^{N}$ has eigenvalues $\lambda_{k}$ and degeneracy $\Gamma_{k}$ given by

$$
\lambda_{k}=k(k+N-1), \quad \Gamma_{k}=\left(\begin{array}{c}
N+k \\
k
\end{array}\right)-\left(\begin{array}{c}
N+k-2 \\
k-2
\end{array}\right), \quad k=0,1,2, \ldots
$$

This means that the zeta function $\zeta_{\Delta}(s)$ is given by

$$
\begin{aligned}
\zeta_{\Delta}(s) & =\sum_{k=0}^{\infty} \frac{\Gamma_{k}}{\left\{k(k+N-1)+(N-1)^{2} / 4\right\}^{s}} \\
& =\sum_{k=0}^{\infty} \frac{\Gamma_{k}}{\{k+(N-1) / 2\}^{2 s}},
\end{aligned}
$$

where the reader will notice that we have completed the square in the denominator on the right-hand side. The expression for the degeneracy must be made more explicit: we have

$$
\begin{aligned}
\Gamma_{k} & =\left(\begin{array}{c}
N+k \\
k
\end{array}\right)-\left(\begin{array}{c}
N+k-2 \\
k-2
\end{array}\right)=\left(\begin{array}{c}
N+k \\
N
\end{array}\right)-\left(\begin{array}{c}
N+k-2 \\
N
\end{array}\right) \\
& =\frac{(k+N)(k+N-1) \ldots(k+1)}{N !}-\frac{(k+N-2)(k+N-3) \ldots(k+1) k(k-1)}{N !} \\
& =\frac{(k+N-2) \ldots(k+1)}{N !}\{(k+N)(k+N-1)-k(k-1)\} \\
& =\frac{(k+N-2) \ldots(k+1)}{N !}\{2 N k+N(N-1)\} \\
& =\frac{2}{(N-1) !}(k+N-2) \ldots(k+1)\{k+(N-1) / 2\} .
\end{aligned}
$$

With this expression for the degeneracy we can write $\zeta_{\Delta}(s)$ as

$$
\begin{aligned}
\zeta_{\Delta}(s) & =\frac{2}{(N-1) !} \sum_{k=0}^{\infty} \frac{(k+N-2) \ldots(k+1)\{k+(N-1) / 2\}}{\{k+(N-1) / 2\}^{2 s}} \\
& =\frac{2}{(N-1) !} \sum_{k=0}^{\infty} \frac{(k+N-2) \ldots(k+1)}{\{k+(N-1) / 2\}^{2 s-1}} .
\end{aligned}
$$


Now since $N$ is odd we write

$$
N=2 m+1
$$

and obtain

$$
\zeta_{\Delta}(s)=\frac{2}{(2 m) !} \sum_{k=0}^{\infty} \frac{(k+2 m-1) \ldots(k+1)}{(k+m)^{2 s-1}} .
$$

Next we use the variable

$$
k^{\prime}=k+m
$$

so that

$$
\begin{aligned}
(k+2 m-1) \ldots(k+1)= & \left(k^{\prime}+m-1\right)\left(k^{\prime}+m-2\right) \ldots \\
& \times\left(k^{\prime}+1\right) k^{\prime}\left(k^{\prime}-1\right) \ldots\left(k^{\prime}-m+1\right) \\
= & k^{\prime} \prod_{i=1}^{m-1}\left\{\left(k^{\prime}\right)^{2}-i^{2}\right\} .
\end{aligned}
$$

Using this factorised expression we find that

$$
\zeta_{\Delta}(s)=\frac{2}{(2 m) !} \sum_{k=m}^{\infty} \frac{\prod_{i=1}^{m-1}\left\{k^{2}-i^{2}\right\}}{k^{2 s-2}} .
$$

But since the product expression on the right-hand side of (3.15) is a polynomial in $k^{\prime}$ we can expand it as (we also drop the prime)

$$
\prod_{i=1}^{m-1}\left\{k^{2}-i^{2}\right\}=\sum_{j=0}^{m-1} E_{j} k^{2 j}
$$

for suitable coefficients $E_{j}$. This in turn allows us to write the zeta function as

$$
\zeta_{\Delta}(s)=\frac{2}{(2 m) !} \sum_{k=m}^{\infty} \sum_{j=0}^{m-1} \frac{E_{j}}{k^{2 s-2 j-2}} .
$$

The final stage in the calculation of the Casimir energy is to peruse the right-hand side of (3.18) and see that it is closely related to the classical Riemann zeta function $\zeta(s)$ which we recall is defined by

$$
\zeta(s)=\sum_{k=1}^{\infty} \frac{1}{k^{s}} .
$$

If we start the summation defining $\zeta(s)$ at $m$ rather than at unity then we obtain a function which we shall denote by $\zeta^{m}(s)$. Clearly we have

$$
\zeta^{m}(s)=\sum_{k=m}^{\infty} \frac{1}{k^{s}} \quad \text { and also } \quad \zeta^{1}(s) \equiv \zeta(s) .
$$

Using the definition of $\zeta^{m}(s)$ in (3.18) we find easily that

$$
\zeta_{\Delta}(s)=\frac{2}{(2 m) !} \sum_{j=0}^{m-1} E_{j} \zeta^{m}(2 s-2 j-2) .
$$


But $\zeta^{m}(s)$ may easily be related to the classical zeta function since we can write

$$
\zeta(s)=\sum_{k=1}^{m-1} \frac{1}{k^{s}}+\sum_{k=m}^{\infty} \frac{1}{k^{s}} \Rightarrow \zeta^{m}(s)=\zeta(s)-\sum_{k=1}^{m-1} \frac{1}{k^{s}} .
$$

Hence our completely explicit expression for $\zeta_{\Delta}(s)$ is therefore

$$
\zeta_{\Delta}(s)=\frac{2}{(2 m) !} \sum_{j=0}^{m-1} E_{j}\left\{\zeta(2 s-2 j-2)-\sum_{k=1}^{m-1} \frac{1}{k^{2 s-2 j-2}}\right\}
$$

and, since the Riemann zeta functions $\zeta(2 s-2 j-2)$ on the right-hand side all have meromorphic analytic continuations, we have achieved our goal of constructing an analytic continuation of $\zeta_{\Delta}(s)$.

The poles of $\zeta_{\Delta}(s)$ all come from the $\zeta(2 s-2 j-2)$ terms: the zeta function $\zeta(s)$ has only one pole this being at $s=1$ with residue 1 . Thus the poles of $\zeta_{\Delta}(s)$ are all simple and occur at the values were

$$
\begin{aligned}
2 s-2 j-2 & =1, \quad j=0, \ldots,(m-1) \\
\Rightarrow s & =j+3 / 2, \quad j=0, \ldots,(m-1) \quad \text { recall } N=2 m+1 \\
\text { or } \quad s & =N / 2-n, \quad n=0,1, \ldots,(N / 2-3 / 2) .
\end{aligned}
$$

The pole locations listed in (3.24) above are in complete agreement with the general theory of Sect. 2 which allows for poles at

$$
s=N / 2-n, \quad n=0,1, \ldots
$$

However we note the vital point that in (3.24) there is no pole at $s=-1 / 2$ (though it is generically present) and thus the Casimir energy is finite as promised. obtain

Passing to the Casimir energy itself means setting $s=-1 / 2$ and, doing this, we

$$
\begin{aligned}
\zeta_{\Delta}(-1 / 2) & =\frac{2}{(2 m) !} \sum_{j=0}^{m-1} E_{j}\left\{\zeta(-2 j-3)-\sum_{k=1}^{m-1} k^{2 j+3}\right\} \\
& =\frac{2}{(2 m) !}\left\{\sum_{j=0}^{m-1} E_{j} \zeta(-2 j-3)-\sum_{j=0}^{m-1} E_{j} \sum_{k=1}^{m-1} k^{2 j+3}\right\} .
\end{aligned}
$$

But the second term on the right-hand side of (3.25) vanishes for interchanging the finite summations we have

$$
\begin{aligned}
\sum_{j=0}^{m-1} E_{j} \sum_{k=1}^{m-1} k^{2 j+3} & =\sum_{k=1}^{m-1} \sum_{j=0}^{m-1} E_{j} k^{2 j+3} \\
& =\sum_{k=1}^{m-1} k^{3} \sum_{j=0}^{m-1} E_{j} k^{2 j} \\
& =\sum_{k=1}^{m-1} k^{3} \prod_{i=1}^{m-1}\left\{k^{2}-i^{2}\right\}, \quad \text { using Def. (3.17) } \\
& =0, \text { since one term in the product is always zero }
\end{aligned}
$$

Thus for the Casimir energy we have the simple expression

$$
\mathscr{E}=\frac{\hbar}{(2 m) !} \sum_{j=0}^{m-1} E_{j} \zeta(-2 j-3), \quad N=2 m+1 .
$$


Before commenting on $\mathscr{E}$ for general (odd) values of $N$ we give $\mathscr{E}$ for a few specific values of $N$. We find that

$$
\mathscr{E}=\left\{\begin{array}{cc}
\hbar / 240, & N=3 \\
-31 \hbar / 60480, & N=5 \\
289 \hbar / 604800, & N=7 \\
\vdots & \vdots
\end{array} .\right.
$$

We note that the sign of $\mathscr{E}$ alternates as $N$ increases.

In fact we shall now prove that, for a general value of $N=2 m+1$, the sign of $\mathscr{E}$ alternates as $m$ increases. The proof follows from two simple facts:

(a) The signs in the sequence $\{\zeta(-3), \zeta(-5), \ldots\}$ alternate because $\zeta(s)$ has simple zeroes at $\{-2,-4, \ldots\}$.

(b) The signs in the sequence $\left\{E_{0}, E_{1}, \ldots\right\}$ alternate as is transparent from their definition (3.17); the sequence will start with a positive or a negative sign depending on the parity of $m$.

A moment's thought now shows that the terms $E_{j} \zeta(-2 j-3)$ of (3.27) are (depending on the parity of $m$ ) either all negative or all positive. Thus we have indeed proved the sign of $\mathscr{E}$ alternates as $m$ increases. In particular, in contrast to what happens in even dimensions, $\mathscr{E}$ is never zero. We now turn to the even dimensional case.

\section{The Casimir Energy of Even Dimensional Spheres}

We can start with the expression (3.12) which is valid for even and odd dimensions so that we have

$$
\zeta_{\Delta}(s)=\frac{2}{(N-1) !} \sum_{k=0}^{\infty} \frac{(k+N-2) \ldots(k+1)}{\{k+(N-1) / 2\}^{2 s-1}} .
$$

With $N=2 m$ this becomes

$$
\zeta_{\Delta}(s)=\frac{2}{(2 m-1) !} \sum_{k=0}^{\infty} \frac{(k+2 m-2) \ldots(k+1)}{(k+m-1 / 2)^{2 s-1}} .
$$

Now using a similar technique to that employed in odd dimensions we define

$$
\begin{aligned}
\bar{k}=k+(m-1 / 2) & \\
\zeta_{\Delta}(s) & =\frac{2}{(2 m-1) !} \sum_{\bar{k}=m-1 / 2}^{\infty} \frac{\{\bar{k}+(2 m-3) / 2\}\{\bar{k}+(2 m-5) / 2\} \ldots\{\bar{k}-(2 m-3) / 2\}}{\bar{k}^{2 s-1}} \\
& =\frac{2}{(2 m-1) !} \sum_{\bar{k}=m-1 / 2}^{\infty} \frac{\prod_{i=1}^{m-1}\left\{\bar{k}^{2}-(2 i-1)^{2} / 2^{2}\right\}}{\bar{k}^{2 s-1}} .
\end{aligned}
$$

To deal with the product we define the coefficients $D_{j}$ by writing

$$
\prod_{i=1}^{m-1}\left\{\overline{k^{2}}-(2 i-1)^{2} / 2^{2}\right\}=\sum_{j=0}^{m-1} D_{j} \bar{k}^{2 j},
$$


and then we obtain

$$
\begin{aligned}
\zeta_{\Delta}(s) & =\frac{1}{(2 m-1) !} \sum_{\bar{k}=m-1 / 2}^{\infty} \sum_{j=0}^{m-1} \frac{D_{j}}{\bar{k}^{2 s-2 j-1}} \\
& =\frac{2}{(2 m-1) !} \sum_{j=0}^{m-1} D_{j} \sum_{\bar{k}=m-1 / 2}^{\infty} \frac{1}{\bar{k}^{2 s-2 j-1}} \\
& =\frac{2}{(2 m-1) !} \sum_{j=0}^{m-1} D_{j} \zeta^{m-1}(2 s-2 j-1,1 / 2),
\end{aligned}
$$

where the functions $\zeta^{m-1}(2 s-2 j-1,1 / 2)$ have a similar definition to the functions $\zeta^{m}(s)$ : more precisely $\zeta^{m}(s, 1 / 2)$ is defined by

$$
\zeta^{m}(s, 1 / 2)=\sum_{k=m}^{\infty} \frac{1}{(k+1 / 2)^{s}} .
$$

The functions $\zeta^{m}(s, 1 / 2)$ are in turn simply related to the Hurwicz zeta function $\zeta(s, 1 / 2)$ whose definition is just

$$
\begin{aligned}
\zeta(s, 1 / 2) & =\sum_{k=0}^{\infty} \frac{1}{(k+1 / 2)^{s}} \\
\Rightarrow \zeta^{m}(s, 1 / 2) & =\zeta(s, 1 / 2)-\sum_{i=1}^{m} \frac{1}{\{(2 i-1) / 2\}^{s}} .
\end{aligned}
$$

After using these definitions the expression we obtain for $\zeta_{\Delta}(s)$ is

$$
\zeta_{\Delta}(s)=\frac{2}{(2 m-1) !} \sum_{j=0}^{m-1} D_{j}\left\{\zeta(2 s-2 j-1,1 / 2)-\sum_{i=1}^{m-1} \frac{1}{\{(2 i-1) / 2\}^{2 s-2 j-1}}\right\} .
$$

Now the Hurwicz zeta function $\zeta(s, 1 / 2)$ can be expressed in terms of the Riemann zeta $^{2}$ function by the equation

$$
\zeta(s, 1 / 2)=\left(2^{s}-1\right) \zeta(s) .
$$

The result of using this fact is the equality

$$
\begin{aligned}
\zeta_{\Delta}(s)= & \frac{2}{(2 m-1) !} \sum_{j=0}^{m-1} D_{j}\left\{\left(2^{2 s-2 j-1}-1\right) \zeta(2 s-2 j-1)\right. \\
& \left.-\sum_{i=1}^{m-1} \frac{1}{\{(2 i-1) / 2\}^{2 s-2 j-1}}\right\} .
\end{aligned}
$$

This then is the analytic continuation of $\zeta_{\Delta}(s)$ for even dimensions and we see that, as guaranteed by the general theory for even dimensions, there is no pole at $s=-1 / 2$; passing to this value and supplying the factor $\hbar / 2$ we get the Casimir energy which is given by

$$
\mathscr{E}=\frac{\hbar}{(2 m-1) !} \sum_{j=0}^{m-1} D_{j}\left\{\left(2^{-2 j-2}-1\right) \zeta(-2 j-2)-\sum_{i=1}^{m-1} \frac{1}{\{(2 i-1) / 2\}^{-2 j-2}}\right\} .
$$

\footnotetext{
${ }^{2}$ Some useful references on the Riemann zeta function are [13-15]
} 
But the second term on the right-hand side of (4.12) is zero in an analogous way to the result of (3.26); we have

$$
\begin{aligned}
\sum_{j=0}^{m-1} D_{j} \sum_{i=1}^{m-1} \frac{1}{\{(2 i-1) / 2\}^{-2 j-2}}= & \sum_{i=1}^{m-1} \sum_{j=0}^{m-1} D_{j} \frac{1}{\{(2 i-1) / 2\}^{-2 j-2}} \\
= & \sum_{i=1}^{m-1}\{(2 i-1) / 2\}^{2} \sum_{j=0}^{m-1} D_{j}\{(2 i-1) / 2\}^{2 j} \\
= & \sum_{i=1}^{m-1}\{(2 i-1) / 2\}^{2} \prod_{j=1}^{m-1}\left[\{(2 i-1) / 2\}^{2}\right. \\
& \left.-\{(2 j-1) / 2\}^{2}\right] \\
= & 0
\end{aligned}
$$

where of course we used (4.5). This means that

$$
\mathscr{E}=\frac{\hbar}{(2 m-1) !} \sum_{j=0}^{m-1} D_{j}\left(2^{-2 j-2}-1\right) \zeta(-2 j-2) .
$$

But

$$
\zeta(s)=0, \quad s=-2,-4,-6, \ldots,
$$

and it follows immediately that

$$
\mathscr{E}=0, \text { for } N=2 m
$$

as claimed in the introduction.

\section{The Casimir Energy for $\xi \neq(N-1) / 4 N$}

Now we would like to turn to a matter alluded to in the introduction namely the value of the parameter $\xi$. Unlike the odd dimensional case, in even dimensions the parameter $\xi$ can be varied without encountering a pole in the zeta function. This means that we can consider the Casimir energy $\mathscr{E}$ as a function of $\xi$. More precisely we write

$$
\mathscr{E} \equiv \mathscr{E}(\lambda)=\frac{\hbar}{2} \zeta_{\Delta}(-1 / 2), \quad \text { with }\left\{\begin{array}{l}
\Delta=-\nabla^{2}+\lambda I \\
\lambda=\xi N(N-1), \quad N=2 m
\end{array}\right.
$$

Thus far we have found that

$$
\mathscr{E}(\lambda)=0, \quad \text { when } \lambda=\frac{(N-1)^{2}}{4}, \quad N=2 m
$$

We shall now see that

$$
\left.\frac{d \mathscr{E}(\lambda)}{d \lambda}\right|_{\lambda=\frac{(N-1)^{2}}{4}}=0, \quad N=2 m
$$

so that $\lambda=(N-1)^{2} / 4$ is a critical point. In fact as $N$ increases this critical point strictly alternates between being a maximum and a minimum. For the lowest value, 
$N=2$ we have a maximum. The verification of this is a routine application of the methods developed above; the key point is to realise that

$$
\frac{d \zeta_{\Delta+\lambda I}(s)}{d \lambda}=-s \zeta_{\Delta+\lambda I}(s+1)
$$

We can give the formula that results from this critical point calculation and quote some actual values for specific $N$. For the second derivative we find that

$$
\begin{aligned}
\left.\frac{d^{2} \mathscr{E}(\lambda)}{d \lambda^{2}}\right|_{\lambda=\frac{(N-1)^{2}}{4}} & =-\frac{\hbar}{2^{2}} \frac{1}{(2 m-1) !} \sum_{j=0}^{m-1} D_{j}\left(2^{2-2 j}-1\right) \zeta(2-2 j) \\
& =-\frac{\hbar}{2^{2}} \frac{1}{(2 m-1) !}\left(2^{2}-1\right) D_{0} \zeta(2) \\
& =(-1)^{m} \frac{3 \hbar \pi^{2}}{24(2 m-1) !} \prod_{i=1}^{m-1}\{(2 i-1) / 2\}^{2}
\end{aligned}
$$

and for various $N$ this gives the table

$$
\left.\frac{d^{2} \mathscr{E}(\lambda)}{d \lambda^{2}}\right|_{\lambda=\frac{(N-1)^{2}}{4}}=\left\{\begin{array}{cc}
-\hbar \pi^{2} / 8, & N=2 \\
+\hbar \pi^{2} / 192, & N=4 \\
\vdots & \vdots
\end{array}\right.
$$

This result about the criticality of $\mathscr{E}(\lambda)$ at the "conformal value" $\lambda=(N-1)^{2} / 4$ (means that we have now extended our knowledge about the Casimir energy to a neighbourhood containing this special value. Denoting the size of such a neighbourhood by $\varepsilon$ we can summarise this in the statement

$$
\mathscr{E}(\lambda)=\left\{\begin{aligned}
0, & \text { if } \lambda=(N-1)^{2} / 4 \\
>0, & \text { for } 0<\left|\lambda-(N-1)^{2} / 4\right|<\varepsilon \text { and } N=2,6, \ldots \\
<0, & \text { for } 0<\left|\lambda-(N-1)^{2} / 4\right|<\varepsilon \text { and } N=4,8, \ldots
\end{aligned}\right.
$$

We can even move "further away" from the point $\lambda=(N-1)^{2} / 4$ and work out the Casimir energy for $\lambda=0$; this corresponds to the pure Laplacian case where $\Delta \equiv-\nabla^{2}$. We have done this. However, since the eigenvalues are no longer perfect squares, this requires a much more delicate treatment of the analytic continuation. The continuation no longer takes such a compact form and is expressed as a (convergent) infinite series. Fortunately this series can be bounded both from above and below and this enables us to determine the sign of the Casimir energy.

For example, if $N=2$ and $\lambda=0$, the analytic continuation takes the form

$$
\zeta_{\Delta}(s)=\sum_{k=0}^{3} B_{k}(z) \zeta(2 z+k-2)+R(z), \quad \text { where } s=-1 / 2+z .
$$

In the above the $B_{k}(z)$ are known expressions and are finite at the "Casimir energy point" $z=0, R(0)$ is the infinite convergent series referred to above. If we evaluate at $z=0$ then we find that the Casimir energy obeys the bound

$$
-0.3226 \frac{\hbar}{2}<\mathscr{E}(0)<-0.22734 \frac{\hbar}{2}
$$


so that $\mathscr{E}(0)$ is negative and this suggests that there are no zeroes of $\mathscr{E}(\lambda)$ between 0 and $(N-1)^{2} / 4$. We conjecture that this is so.

This more powerful continuation method is also applicable to the case of odd dimensions where it should turn up a pole at $s=-1 / 2$ in $\zeta_{\Delta}(s)$ away from $\lambda=(N-1)^{2} / 4$. This indeed happens and a formula for this residue is obtained: the residue is, in fact, a polynomial in $\lambda$ with a root at $\lambda=(N-1)^{2} / 4$. This latter result is quite gratifying since it agrees exactly with what we calculated from the differential-geometric heat equation formulae earlier, cf. for example, (3.3) and (3.4).

\section{Conclusion}

We have shown that zeta function regularisation can be successfully implemented for the calculation of the Casimir energy of spin zero fields on both even and odd dimensional spheres. This has been done by coupling the scalar curvature to the fields with the parameter $\xi$. For odd dimensional spheres $\xi$ must have discrete values one of which is the special value $(N-1) / 4 N$, which results in the conformally coupled wave equation in $N+1$ dimensions. For even dimensional spheres any value of $\xi$ can be used, though the Casimir energy vanishes for ${ }^{3}$ $\xi=(N-1) / 4 N$. In both cases $\xi=(N-1) / 4 N$ is special, in that the eigenvalues of the $N$-dimensional elliptic operator are perfect squares and the trace anomaly of the $N+1$-dimensional theory vanish for this value only. For odd dimensional spheres it is necessary that the trace anomaly in $N+1$-dimensions vanishes in order that the zeta function can be applied. Knowing the Casimir energy and the trace anomaly allows the calculation of the complete energy-momentum tensor.

For conformal coupling in $N+1$ dimensions the sign of the Casimir energy alternates as $N$ increases in the sequence $-, 0,+, 0,-, 0,+, 0, \ldots$ for $N=1,2$, $3, \ldots$ In even dimensions we can vary $\xi$ and conformal coupling gives an extremum being a maximum for $N=2 \bmod 4$ and a minimum for $N=0 \bmod 4$. This suggests stability (instability) of the theory of $N=0(2) \bmod 4$. For $\xi=0$ upper and lower bounds on the Casimir energy can be obtained for even $N$ and it is conjectured that it never vanishes between $\xi=0$ and $\xi=(N-1) / 4 N$.

Acknowledgement. We are grateful to J.S. Dowker for sending us a preprint with some similar results.

\section{References}

1. Birrell, N.D., Davies, P.C.W.: Quantum fields in curved space. Cambridge: Cambridge University Press 1982

2. Gilkey, P.B.: Invariance theory, the heat equation and the Atiyah-Singer index theorem. Publish or Perish 1984

3. Hawking, S.W.: Zeta function regularisation of path integrals in curved spacetime. Commun. Math. Phys. 55, 133-148 (1977)

4. Dowker, J.S., Banach, R.: Quantum field theory on Clifford-Klein space-times. The effective Lagrangian and vacuum stress-energy tensor. J. Phys. A 11, 2255-2284 (1978)

5. DeWitt, B.: The dynamical theory of groups and fields. In: Relativity, Groups and Topology DeWitt, B., DeWitt, C. (eds.). London: Gordon and Breach 1965

${ }^{3}$ Recall that $\lambda=\xi N(N-1)$ so that $\xi=(N-1) / 4 N$ corresponds to $\lambda=(N-1)^{2} / 4$ 
6. Candelas, P., Weinberg, S.: Nucl. Phys. B237, 397 (1984)

7. Birmingham, D., Sen, S.: The Candelas-Weinberg model for spaces $M \times S^{2 N}$. Ann. Phys. 172, 451-482 (1986)

8. Moss, I.G.: Class. Quant. Gravity 6, 759 (1989)

9. McAvity, D.M., Osborn, H.: A DeWitt expansion of the heat kernel for manifolds with a boundary. Class. Quant. Gravity 8, 603-638 (1991)

10. Amsterdamski, P., Berkin, A.L., O'Connor, D.J.: $b_{8}$ "Hamidew" coefficient for a scalar field. Class. Quant. Gravity 6, 1981-1991 (1989)

11. Avramidi, G.: The covariant technique for the calculation of heat kernel asymptotic expansion. Phys. Lett. B238, 92-97 (1990)

12. Avramidi, G.: A covariant technique for the calculation of the one loop effective action. Nucl. Phys. B355, 712-754 (1991)

13. Patterson, S.J.: An introduction to the theory of the Riemann zeta function. Cambridge: Cambridge University Press 1988

14. Ivic, A.: The Riemann zeta-function: The theory of the Riemann zeta-function with applications. New York: Wiley 1985

15. Titchmarsh, E.C.: The theory of the Riemann zeta-function. Oxford: Oxford University Press 1951

Communicated by A. Connes 
